\title{
Severity of Spatial Learning Impairment in Aging: Development of a Learning Index for Performance in the Morris Water Maze
}

\author{
Michela Gallagher, \\ Department of Psychology, University of North Carolina at Chapel Hill \\ Rebecca D. Burwell, and \\ Department of Psychology, University of North Carolina at Chapel Hill \\ Margaret Burchinal \\ Department of Psychology, University of North Carolina at Chapel Hill
}

\begin{abstract}
The Morris water maze task was originally designed to assess the rat's ability to learn to navigate to a specific location in a relatively large spatial environment. This article describes new measures that provide information about the spatial distribution of the rat's search during both training and probe trial performance. The basic new measure optimizes the use of computer tracking to identify the rat's position with respect to the target location. This proximity measure was found to be highly sensitive to age-related impairment in an assessment of young and aged male Long-Evans rats. Also described is the development of a learning index that provides a continuous, graded measure of the severity of age-related impairment in the task. An index of this type should be useful in correlational analyses with other neurobiological or behavioral measures for the study of individual differences in functional/biological decline in aging.
\end{abstract}

A test of spatial learning introduced over a decade ago by Richard Morris (Morris, 1981; Morris, Garrud, Rawlins, \& O'Keefe, 1982) has become widely used in neurobiological studies of limbic-cortical function and in the characterization of cognitive decline in aged rats. Originally devised as a test of "place" learning, the water maze task permits a number of variations that have made it useful for isolating cognitive deficits apart from nonspecific impairments in sensorimotor function. The purpose of the present article is to describe some new methods for behavioral analysis that were developed to supplement those measures commonly used for the water maze. As a framework for presenting the new methods, the following introduction provides a brief discussion of the traditional analysis of performance in this task.

\section{Measures Traditionally Used for Behavioral Analysis in the Water Maze}

The Morris maze apparatus consists of a large, circular pool filled with water that has been made opaque through the addition of powdered milk or some other substance. In the typical "hidden-platform" version of the task, rats are trained to find a camouflaged escape platform

Correspondence concerning this article should be addressed to Michela, Gallagher, Department of Psychology, CB\# 3270, Davie Hall, Chapel Hill, North Carolina 27599-3270. 
that is positioned just below the water surface. The location of this platform remains constant from trial to trial. Because there are no local cues that mark the position of the platform, the rat's ability to locate it efficiently depends on the rat's use of a configuration of extramaze cues surrounding the pool. Indeed, rats can learn to swim directly to the escape platform within relatively few training trials from any of a number of start locations at the perimeter of the pool. Learning is reflected in shorter latencies to escape and by decreases in the length of the path that the rat traverses in reaching the platform.

Although optimal performance in the hidden-platform version of the task requires that rats learn the location of the platform, it was recognized early on that this was not the only learning strategy that could yield improved performance. To illustrate an alternate strategy used by rats to locate the platform, Figure 1 shows data collected on probe trials in a study of rats with hippocampal neurotoxic lesions. The records show the search strategy of trained animals during a fixed interval when the platform was unavailable for escape. The rat from the control group (shown in A. on the left) directs its search predominantly to the area of the maze where the platform was located during training. In contrast, the rat with hippocampal damage swims in concentric circles at a relatively fixed distance from the wall of the pool (shown in B. on the right). In addition to observations in several reports on the effects of hippocampal damage (DiMattia \& Kesner, 1988; Gallagher \& Holland, 1992; Morris, Schenk, Tweedie, \& Jarrard, 1990), it has been noted that some aged rats also use a circling strategy (Gallagher, 1991) and that young rats can be induced to do so when the platform location is pseudorandomly varied among the four maze quadrants during training (Devan, Blank, \& Petri, 1992). This circling strategy is less efficient than learning the location of the platform but can support considerably better escape performance than a random search when the platform is available on training trials. Thus decreased latencies and path lengths across a series of training trials are not necessarily an indication of place learning. The relatively imprecise nature of these measures in assessing place learning emphasizes the importance of probe trials for analyzing the learning strategy used in this task.

An additional shortcoming of the traditional measures used for training trials is illustrated in Figure 2. Two training trial records are shown. In the record on the left, the path is widely distributed, with little focused search in any particular region of the maze. In the record on the right, the search is focused more in proximity to the escape platform. The escape latencies are virtually identical for these two trials. Computing the path length to reach the escape platform also does not distinguish performance because path length per se does not contain information about the spatial distribution of the rat's search relative to the goal. Indeed, the path length to locate the escape platform is slightly shorter in A $(573 \mathrm{~cm})$ than in B $(590 \mathrm{~cm})$. However, performance on the two trials differs quite markedly if the animal's location is used to compute distance from the goal during the trial: By the method described in the caption to Figure 2, the cumulative distance from the goal during the search in Trial A is $1,835 \mathrm{~cm}$, whereas it is $781 \mathrm{~cm}$ for Trial B.

Using a measure of proximity to the goal can also be advantageous in the analysis of probe trial performance. The measures commonly used to assess performance on probe trials are designed to reflect the spatial bias of an animal's search pattern. These typically include either the percentage of time that is spent or the percentage of the total path length during 
the search that is traversed within the training quadrant and, as a second measure, so-called platform crossings. A platform crossing occurs when the animal's path traverses the precise location where the escape platform was positioned during training. Figure 3 shows three sample paths from probe trials. The accuracy of the animal's search relative to the escape platform location differs substantially in these three records. However, each of the measures commonly used to analyze probe trials is inadequate to resolve the differences in search accuracy that are evident in this illustration. For example, roughly $50 \%$ of the total path length was located in the training quadrant in both $\mathrm{A}$ and $\mathrm{B}$, which indicates comparable performance by this measure on these trials. The platform-crossing measure distinguishes $\mathrm{A}$ from $\mathrm{B}$ but does not distinguish the performance shown in B from that in $\mathrm{C}$, despite a clear difference in the accuracy of the search on these two trials. An alternative method for analysis of these data is to compute the rat's proximity to the escape platform location over the course of the search. Distance to the goal differs substantially for the three records, averaging $36.9 \mathrm{~cm}$ in A, $50.7 \mathrm{~cm}$ in B, and $75.2 \mathrm{~cm}$ in C. In essence, this new method integrates the types of information normally provided with traditional measures. In addition, the proximity measure provides other advantages: It can be either averaged over the duration of the probe trial, to reflect the entire distribution of the search during the trial, or analyzed in smaller segments of the trial. In the latter case, the measure permits a more fine-grained analysis of behavior over time, for example, to assess extinction during a lengthy probe trial.

This study describes the application of new proximity measures to the behavioral analysis of learning in the water maze task. We did this by comparing proximity measures with traditional measures in an assessment of the performance of young and aged rats. A second purpose of the study was to develop a learning index based on the proximity measure for use in characterizing individual differences in the effects of aging on spatial learning ability. The need for such an index is based on the previously discussed limitations of customary analysis and on certain special features of the effects of aging on spatial learning ability, described next.

Our research and similar studies conducted in a number of other laboratories have shown that the water maze task is sensitive to age-related learning impairment but that striking individual differences are apparent in aged animals (Gage, Dunnett, \& Björklund, 1984; Gallagher \& Burwell, 1989; Rapp, Rosenberg, \& Gallagher, 1987). Thus some aged rats appear to learn the spatial task as proficiently as young animals, whereas other aged rats exhibit marked impairment. Because probe trial data are essential for determining whether performance is based on a search strategy similar to that normally used by young rats, much of our previous research used a protocol in which brief probe trials were interpolated during the course of training. In numerous studies we found that young rats typically reached a criterion performance on probe trials interpolated after 10-15 training trials, whereas fewer than half of the aged rats performed in a comparable manner. Figure 4 illustrates this finding with composite results from a number of previous studies conducted in our laboratory. We used these data to classify aged rats into subgroups designated as unimpaired and impaired in order to examine whether brain aging distinguishes those rats with spatial learning deficits from their aged cohorts with preserved cognitive function. The method of subgrouping aged rats, however, has certain liabilities, as discussed elsewhere (Olton \& Markowska, 1992). To capture the variability in agerelated spatial learning impairment for correlational analyses in 
neurobiological studies, other investigators have selected some composite measure of performance during training trials, for example, mean latency or path length (e.g., see Fischer, Gage, \& Björklund, 1989). These measures, however, may not reflect the severity of spatial learning impairment unconfounded by alternate learning strategies, as discussed in the introduction. Therefore, we developed a learning index in this study to provide a graded measure of place learning impairment for individual aged rats. This type of index could also be used to address other neurobiological issues, for example, whether the severity of behavioral impairment is systematically related to lesion size or to the extent of neurochemical depletion in studies of young animals.

\section{Method}

Subjects

Behavioral results from 96 young (4-6 months) and 128 aged (25-27 months) pathogen-free Long-Evans male rats are presented. The aged subjects were obtained as retired breeders at 8-9 months of age (Charles River Laboratories, Raleigh, NC). The young rats were obtained at 90 days of age from the same source and entered the experiment at 4-6 months of age. The young and aged rats were behaviorally tested in replications that used 6-12 young rats and 10-18 aged rats during the months spanning January 1, 1991 through April 30, 1992. Routine screening for viral antibodies and necropsies at the time of sacrifice were performed to ensure the health of aged subjects. Prior to data analysis, a number of aged animals ( $\mathrm{n}=$ 14) were excluded on the basis of necrospy findings (pituitary tumors, etc.). Tests for viral antibodies were routinely negative, which confirmed the pathogen-free status of the animals used in the study.

\section{Apparatus}

The water maze consisted of a circular tank (diameter $=1.83 \mathrm{~m}$; height $=0.58 \mathrm{~m}$ ). It was filled with tepid water $\left(27^{\circ} \mathrm{C}\right)$ that was made opaque by the addition of powdered milk $(0.9$ $\mathrm{kg}$ ). A white escape platform (height $=34.5 \mathrm{~cm}$ ) was located $1 \mathrm{~cm}$ below the water surface near the center of one of the four quadrants of the maze. This platform could be retracted to the bottom of the tank or raised to its normal position from outside the maze during behavioral testing. A second platform (height $=37.5 \mathrm{~cm}$ ) with its surface painted black was elevated $2 \mathrm{~cm}$ above the water surface during cue training. The maze was surrounded by white curtains with patterns affixed to provide a configuration of spatial cues. Data were analyzed by a video tracking system (HVS Image Analyzing VP-112) and an IBM PC computer with software developed by Richard Baker of HVS Imaging.

\section{Procedure}

In the present study, rats were trained with the same protocol used in our previous research. An analysis of the results done with conventional measures was compared with an analysis based on proximity measures that reflected the spatial distribution of the animal's search relative to the goal.

Rats received three trials per day for 12 consecutive days; a 60-s intertrial interval was used. On each training trial, an animal was released in the maze from one of four equally spaced 
starting positions around the perimeter of the pool. The starting position varied from trial to trial and thus precluded the effective use of a response strategy (e.g., always turning left from the start location to locate the escape platform). If an animal did not locate the escape platform within $90 \mathrm{~s}$ on any trial, the experimenter placed the animal on the platform, where it remained for $30 \mathrm{~s}$. Every sixth trial consisted of a probe trial to assess the development of spatial bias in the maze. During these trials, the animals swam with the platform retracted to the bottom of the pool for $30 \mathrm{~s}$, at which time the platform was raised to its normal position for completion of an escape trial. At the completion of the protocol with the hidden platform, rats in a subset of the replications were assessed for cue learning with the visible platform. The location of this platform varied from trial to trial in a single session of six training trials.

For analysis of spatial learning, measures of path length and latency to escape were analyzed using average performance for blocks of five training trials. Probe trial data were assessed for acquisition of criterion performance, that is, at least 35\% of the duration of the probe trial spent in the training quadrant and at least two platform crossings. Analysis of cue training performance was based on latency and path length to escape. These analyses thus used traditional measures for behavioral assessment in the water maze task.

The proximity of the animal's position with respect to the goal was used to provide several new analyses of training trial and probe trial performance. The proximity measure was obtained by sampling the position of the animal in the maze (10 times per second) to provide a record of its distance from the escape platform in 1-s averages. For both probe trials and training trials, a correction procedure was implemented so that trial performance was relatively unbiased by differences in distance to the goal from the various start locations at the perimeter of the pool. In making this correction we calculated the average swimming speed for each trial (path length/latency). Then the amount of time required to swim to the goal at that speed from the start location used on the trial was removed from the record prior to computing trial performance; that is, cumulative distance was used on training trials and average distance from the goal was used on probe trials. By this method, scores obtained with the proximity measure are designed to reflect search error; they represent deviations from an optimal search, that is, from a direct path to the goal.

The use of proximity measures was first introduced in this laboratory for analyzing probe trial performance and was later adapted in the course of this study for the analysis of training trials. Thus, training trial data are presented for a subset of the animals (young, $\mathrm{n}=64$; aged, $\mathrm{n}=62$ ) included in the probe trial analysis. In addition, cue training data are presented for only a subset of the replications included in this study (young, $n=47$; aged, $n=45$ ) that received this protocol at the end of place training.

\section{Results}

\section{Behavioral Analysis Using Traditional Measures}

As in our earlier work, the criterion adopted for analysis of the search strategy during probe trials included both the percentage of the path length that was in the training quadrant and platformcrossing measures. The results of this study are highly similar to those observed in 
previous research that used the same protocol. With few exceptions, young rats reliably achieved criterion by the third probe trial (Figure 5). Approximately half of the aged rats achieved criterion by this point in training. Statistical analysis (Kolmogorov-Smirnov [KS] test) revealed that there was a significant age-related impairment in acquisition of criterion performance $(\mathrm{KS}=3.09, \mathrm{p}<.0001)$. Analysis of the traditional measures of performance on place training trials also revealed an age difference. Aged rats were impaired, as reflected in both path length, $\mathrm{F}(1,124)=6.59, \mathrm{p}<.01$, and latency, $\mathrm{F}(1,124)=41.60, \mathrm{p}<.0001$, measures. In contrast to these effects of age, there was no significant difference between groups for either latency or path length during cue training. The latency and path length measures averaged across cue training trials for the young group (8.3 $0.87 \mathrm{~s}$ and 18112.8 $\mathrm{cm})$ were highly comparable to those for the aged group $(9.61 .2 \mathrm{~s}$ and $17813.3 \mathrm{~cm})$.

\section{Behavioral Analysis Using a Proximity Measure}

The newly developed proximity measure was used to analyze both place training trials and probe trial performance. Figure 6 shows the performance of young rats during training trials and compares the customary path length measure with a new measure based on proximity to the platform that is referred to as search error. As described in the Method section, search error represents the corrected cumulative distance from the platform. Although numerical values on the two measures are in the same range at the end of training (averaging $181 \mathrm{~cm}$ and $225 \mathrm{~cm}$ for path length and search error, respectively), the magnitude of change from the initial level of performance is much greater for search error, $\mathrm{F}(95,59)=95.6, \mathrm{p}<.0001$. The amount of change was significantly greater for search error than for path length in all adjacent blocks ( $\mathrm{p}<.005$ ), with the exception of the change from Block 5 to Block 6 ( $\mathrm{p}<$. 11).

Figure 7 shows the cumulative search error during place training trials for young and aged groups. As with the path length and latency measures, statistical analysis revealed a significant overall age effect, $F(1,124)=30.80, p<.0001$, but there was no reliable Age $X$ Trial Block interaction, $F(5,120)=1.84, p<.11$. Although path length and search error both yield a difference between the performance of young and aged groups, comparison of the magnitude of the age effect from these analyses indicated greater age sensitivity for the search error measure on all but the first block of training trials ( $p<.002$ for Blocks $2-6$ and $p$ $<.10$ for Block 1).

The proximity of the rat's search to the goal was also used to assess performance on probe trials. Proximity provides a measure of the acquisition of a spatial bias by young rats during training, as shown in Figure 8. Proximity measures for the six probe trials are shown along with the same proximity measures for the first $30 \mathrm{~s}$ of the first training trial (indicated as Trial 0), which serve as a baseline. Data are presented for average proximity to the escape platform (i.e., Target) and proximity to the comparable position in the maze located $180^{\circ}$ from the escape platform (i.e., Opposite). The statistical analysis of these data indicated that there were significant main effects for both probe trial, $\mathrm{F}(5,65)=11.00, \mathrm{p}<.0001$, and location (target vs. opposite), $\mathrm{F}(1,69)=557.1, \mathrm{p}<.0001$, as well as a significant Probe Trial $X$ Location interaction, $F(5,65)=19.70, p<.0001$. Analysis of the interaction revealed that proximity to the target location, $\mathrm{F}(5,65)=18.20, \mathrm{p}<.0001$, and to the opposite location, 
$F(5,65)=9.10, p<.0001$, both changed significantly over the course of training. The rapid acquisition of a spatial bias was evident in the results of step-down comparisons for the magnitude of the difference between proximity to the target and to opposite locations across successive probe trials and comparisons of target proximity alone: $\mathrm{p}<.0001$ for comparison of Probe Trials 1 and 2 and $p<.002$ for comparison of Probe Trials 2 and 3. In contrast, no differences were found in comparison of either Probe Trials 3 and 4 or Probe Trials 5 and 6. These results indicate that approximately asymptotic performance was achieved by the fourth probe trial.

An analysis was also done on these data to determine whether proximity to the target platform location showed evidence for extinction over the 30-s probe trial interval, that is, whether there was a tendency for rats to abandon their search toward the end of the trial. In this analysis, the average proximity during seconds $11-20$ of the interval was compared to the mean proximity during the final $10 \mathrm{~s}$ for each probe trial. The middle segment of the interval was selected for this comparison because the correction procedure removed variable portions of the initial segment (depending on swim speed) from calculation of the proximity measure. The analysis of probe trial performance indicated no reliable difference between segments of the interval for Probe Trials 1-5. On the final probe trial, rats searched in closer proximity to the target location during the middle segment than in the final 10-s segment ( $p$ $<.005)$. Thus, rats did not tend to abandon their search toward the end of the probe trial throughout most of the training protocol: The average proximity measure appears to be representative of the spatial distribution of the search unconfounded by any extinction effect on all but the final probe trial, where a pattern that may reflect extinction was found.

Figure 9 shows the average proximity to the target location for young and aged groups on Probe Trials 1-6. As with other measures normally used to characterize learning in this task, acquisition of an accurate search for the platform was more rapid for young rats than for the aged animals. Statistical analysis of these data revealed an overall age difference, $F(1,193)$ 25.14, $\mathrm{p}<.0001$, and a significant Age Probe Trial interaction, $\mathrm{F}(5,189) 5.84, \mathrm{p}<.0001$. There was no significant difference between the age groups at either the first or last probe trial ( $\mathrm{p} .10)$. On all intervening probe trials the performance of the young group was significantly more accurate than that of the aged group $(\mathrm{p}<.001)$, with the exception of Probe Trial 4, for which the difference between age groups approached significance $(\mathrm{p}<$. 06). Step-down comparisons were performed to examine the magnitude of the age effect across successive probe trials. According to this analysis, the age effect significantly increased early in the training protocol (in the comparison of Probe Trials 1 and $2, \mathrm{p}<.05$ ). A further increase in the magnitude of the age difference approached significance in the comparison of Probe Trials 2 and 3 ( $p<.06$ ). From there on, differences across successive probe trials reflected either no reliable change (Probe Trials 4 and 5) or a diminution of the age effect (comparisons between Probe Trials 3 and 4 and between Probe Trials 5 and 6,p $<.02)$.

\section{Development of a Learning Index Using the Proximity Measure}

Many studies of spatial learning in the water maze use a behavioral protocol in which a single probe trial is placed at the end of training. In such studies, analysis of the spatial 
distribution of the search relative to the goal, as described here, might provide a graded measure for characterizing the performance of individual rats. In the study of aged animals, however, impairment can be obscured if extensive training occurs prior to probe trial assessment. As indicated by the results of the present study, the difference in the performance of young and aged rats becomes most pronounced in a relatively early phase of training, because young rats develop an accurate search strategy more rapidly than aged subjects. This was apparent both in the analysis of the proximity measure and in the assessment of criterion performance on probe trials. Thus the impact of aging on spatial learning ability might be represented best by a measure that reflects the rate at which accurate place learning is acquired.

To this end, we used the proximity measure to calculate an index in which probe trials were weighted so as to favor rapid acquisition: Thus, good performance on the sixth probe trial was not treated on the same basis as a comparably good performance on the second probe trial. The weights were empirically derived from the performance of the young rats included in this experiment: For each probe trial, the weight consisted of a multiplier that was the quotient of the young group's mean proximity for that probe trial and the mean proximity for the first probe trial. The set of multipliers for Probe Trials 1-6 was as follows: 1.00, 1.26, $1.43,1.43,1.43$, and 1.43 . Weights were held constant after the third probe trial because young rats achieve asymptotic performance by that point in training. A comparison was then made of an index based on all probe trials (1-6) with one based on performance during earlier probe trials only (1-4 and 2-4). The index based on Trials $2-4$ excluded the first probe trial because an age difference was not evident for that trial or the block of training trials preceding it.

The learning index scores for the young and aged groups are shown in the upper panel of Figure 10. Correlations were high for comparisons among these three types of index scores. For example, the Pearson $r$ was .95 between the index formed on the basis of Probe Trials 14 and that derived from Probe Trials 2-4; an equally high correlation was found between the latter index and that formed from all probe trials $(r=.92)$. These correlations were also equivalent when young and aged rats were considered separately in the analyses. On each index, the aged rats had significantly higher scores than the young rats $(\mathrm{p}<.005)$. We also performed analyses to compare the learning index scores with our prior method of assigning aged rats to impaired and unimpaired subgroups on the basis of their achieving a criterion performance on a single probe trial. As shown in the bottom panel of Figure 10, aged rats designated as impaired by our original criterion have significantly more impairment according to learning index scores than either the young group $(\mathrm{p}<.0001)$ or the subgroup of aged rats previously designated as unimpaired $(\mathrm{p}<.001)$. In contrast, no significant difference between the young group and the aged-unimpaired subgroup was found for any index. As predicted, the sensitivity for detecting differences was somewhat greater for the index formed on the basis of Probe Trials 2-4 (R2 values were .31, .27, and .18 for the indexes for Probe Trials 2-4, 1-4, and 1-6, respectively). Figure 11 shows the distribution of individual scores (index for Probe Trials 2-4) for one sample replication that is representative of each of the replications included in the overall analysis. 
As might be expected, learning index scores discriminate differences in performance during training. To illustrate this point, the data presented in Figure 12 show the young rats compared to two subgroups of aged rats. Those aged rats with index scores at 240 or above on the index computed for Probe Trials 2-4 were designated impaired (Aged-I) and those aged rats with scores on this index below 240 were designated unimpaired (Aged-U). The score used to divide the aged animals into subgroups is approximately two standard deviations from the mean of young performance on the index. This division also corresponds closely to our prior method of subgrouping aged animals on the basis of a probe-trial criterion performance. The data presented in Figure 12 show that place training performance differs markedly for the aged-impaired subgroup relative to both the young and the aged rats. A similar pattern of results is obtained when the latency and path length data are examined for these same groupings. In each case, statistical analysis revealed that the agedimpaired subgroup differed from both the young and the aged-unimpaired groups $(\mathrm{p}<.005)$. In the case of path length and cumulative distance, the aged-unimpaired group did not differ from the young group, but the aged-unimpaired rats had reliably longer escape latencies than their younger counterparts $(\mathrm{p}<.005)$. Contrary to the impression that might be gained from these findings, however, learning index scores were only modestly correlated with training trial measures. When the same index scores that were used to subgroup the aged rats (based on Probes 2-4) were used, correlations with each training trial measure ranged from a very modest .25 (correlation for young rats on index scores and latency) to a high of .59 (correlation for aged rats on index scores and search error). The fact that learning index scores and measures of performance on training trials are not more strongly correlated indicates that these provide somewhat different estimates of the severity of impairment for individual subjects. Compared with measures of performance during training trials, index scores, which are based on performance during probe trials, are likely to provide a more valid estimate of individual differences in place learning ability. As noted earlier in the introduction, training trial measures are likely to reflect alternative learning strategies.

In contrast to the results showing that index scores discriminate performance during place training, these same scores still fail to detect any impairment during cue training (see Figure 13). Analysis of both latency and path length measures for young, aged-impaired, and agedunimpared rats revealed no group differences. In addition, correlations between learning index scores and cue training measures were very low (i.e., not exceeding - 0.21).

\section{Discussion}

The Morris water maze task was originally designed to assess the rat's ability to learn to navigate to a specific location in a relatively large environment. The new measures and methods described in this report were designed to provide information about the spatial distribution of the rat's search during both training and probe trial performance. The basic measure developed for this purpose optimizes the use of computer tracking for identifying the position of the rat with respect to the target location. This proximity measure was used to assess search error on training trials and to determine the spatial accuracy of the search for the platform on probe trials. 
The analysis of search error was based on the spatial distribution of the rat's performance on training trials, information that is not provided by either of the customary measures used in this task, that is, latency and path length. Compared with path length, search error reveals a greater magnitude of change in performance over the course of training and reflects the degree to which rats improve their ability to localize the target location. This measure also proved to be more sensitive to an effect of age on performance than was the traditional path length measure. Although search error provides a method for analyzing training trial performance that may better reflect the accuracy of spatial learning, it is subject to the same criticism discussed earlier with reference to path length and latency data. As with those measures, search error is liable to be confounded by different learning strategies. Animals that achieve relatively good escape performance by using a circling strategy are likely to have less error than animals that randomly search for the platform, and may, therefore, more closely resemble rats that localize the goal using the configuration of cues surrounding the maze. To the extent that this task is intended to assess learning that is based on processing spatial information, probe trial data arguably provide the most valid assessment of this cognitive ability.

Assessment of proximity to the target provides a more efficient method of analysis than do the multiple measures traditionally used to characterize probe trial performance, that is, platform crossings and path length or time in quadrant. In contrast to those measures, the analysis presented in this article can also provide a single measure that reflects the entire search during a probe test rather than a selected portion of the data, which is the case when, for example, the percentage of the path length in the training quadrant is selected as the measure of interest. An analysis of young rat performance that used this measure showed rapid acquisition of improved search accuracy during the interpolated probe trials. Comparison of young and aged groups demonstrated that the effect of age was most pronounced relatively early in training, because aged rats acquired a spatial bias more slowly. Thus, in this study the probe trial analysis using proximity to the target was sensitive to an age-related impairment in spatial learning.

The probe trial proximity data were then used to compute a learning index score that was designed to integrate performance over the course of training. Thus, the index reflects performance on multiple, interpolated probe trials. Although a simple average of performance on these probe trials might be used, our index was also constructed to reflect rate of acquisition. This learning index discriminated individual differences among aged rats that agreed closely with a prior method for subgrouping aged animals on the basis of their achieving criterion performance on a single probe trial. In contrast to the prior subgrouping method, however, the index scores provide a continuous measure of the severity of agerelated impairment. The index formed on the basis of Probe Trials 2-4 appeared to provide the most sensitive index, which was, nonetheless, highly correlated with the index that was based on all probe trials included in the training protocol. There is currently strong interest in determining the systems in the brain in which age-related neurobiological changes are most closely coupled to individual differences in the severity of cognitive decline (for reviews see Collier \& Coleman, 1991; Gallagher, Nagahara, \& Burwell, in press; Rapp \& Amaral, 1992). Learning index scores of the type described here should provide a graded 
measure of spatial learning in the water maze task that is suitable for correlational analyses with other neurobiological or behavioral data.

The characterization of aged rat performance with the methods used in this study supports the use of this task as a model for cognitive impairment. As previously reported, aged LongEvans rats exhibit deficits in the hidden-platform version of the task but not in cue learning (Gallagher \& Burwell, 1989). Although learning index scores readily identified the aged rats that performed most poorly during place training, those animals (aged-impaired) were also unimpaired in the cued version of the task. Moreover, learning index scores were not correlated with measures of cue learning (latency and path length). Intact performance during cue training indicates that deficits in the spatial task are not attributable to general motivation or to sensorimotor deficits, an interpretation that has been applied to a similar pattern of results across the two versions of this task in rats with hippocampal damage (Morris et al., 1982). Not all strains of aged rats yield results comparable to those reported in the present study, however. In particular, albino strains (Sprague-Dawley and Fischer 344 rats) have been found to have age-related impairment in cue learning, and such deficits seem to be pronounced in those aged rats with the most severe spatial learning deficits (Clark, Magnusson, \& Cotman, 1992; Gage \& Björklund, 1986; Markowska et al., 1989). Indeed, Markowska et al. (1989) reported that measures of performance during place and cue training were strongly correlated for aged Sprague-Dawley rats (e.g., $\mathrm{r}=.82$ for $\mathrm{N}=32$ in that study). In such cases it is difficult to interpret deficits in the hidden-platform version of the task as indicative of cognitive loss.

In cases where cue learning is intact, the notion that age-related impairment in spatial learning is not merely due to performance deficits is also bolstered by the fact that at the outset of training in the hidden-platform task, aged rats perform on a par with young animals. This was evident in the present study for the analysis of both search error on training trials (first block of trials) and proximity to the platform during probe trials (first probe trial). Similar results were found when those aged rats that were most impaired in place learning were considered separately. Thus, it appears that some rats become less proficient in learning the information that is required for efficient navigation to a specific location. The new methods described in this article are offered as a sensitive, efficient, and valid approach to the assessment of this age-related cognitive deficit.

\section{Acknowledgments}

Funding: P01-AG009973, F31-MH10185, KO2-MH 100406

\section{References}

Burwell R, Gallagher M. A longitudinal study of reaction time performance in Long-Evans rats. Neurobiology of Aging. 1993; 14:57-64. [PubMed: 8450934]

Clark AS, Magnusson KR, Cotman CW. In vitro autoradiography of hippocampal excitatory amino acid binding in aged Fischer 344 rats: Relationship to performance on the Morris water maze. Behavioral Neuroscience. 1992; 2:324-335.

Collier TJ, Coleman PD. Divergence of biological and chronological aging: Evidence from rodent studies. Neurobiology of Aging. 1991; 12:685-693. [PubMed: 1791906] 
Devan BD, Blank GS, Petri HL. Place navigation in the Morris water task: Effects of reduced platform interval lighting and pseudorandom platform positioning. Psychobiology. 1992; 20:120-126.

DiMattia BD, Kesner RP. Spatial cognitive maps: Differential role of parietal cortex and hippocampal formation. Behavioral Neuroscience. 1988; 102:471-480. [PubMed: 3166721]

Fischer W, Gage FH, Björklund A. Degenerative changes in forebrain cholinergic nuclei correlate with cognitive impairments in aged rats. European Journal of Neuroscience. 1989; 1:34-45. [PubMed: 12106172]

Gage FH, Björklund A. Cholinergic septal grafts into the hippocampal formation improve spatial learning and memory in aged rats by an atropine sensitive mechanism. Journal of Neuroscience. 1986; 6:2837-2847. [PubMed: 3760938]

Gage FH, Dunnett SB, Björklund A. Spatial learning and motor deficits in aged rats. Neurobiology of Aging. 1984; 5:43-48. [PubMed: 6738785]

Gallagher M. Aged rat performance on probe trials. 1991 Unpublished raw data.

Gallagher M, Burwell RD. Relationship of age-related decline across several behavioral domains. Neurobiology of Aging. 1989; 10:691-708. [PubMed: 2628781]

Gallagher M, Holland PC. Preserved configural learning and spatial learning impairment in rats with hippocampal damage. Hippocampus. 1992; 2:81-88. [PubMed: 1308174]

Gallagher, M., Nagahara, A., Burwell, R. Cognition and hippocampal systems in aging: Animal models. In: McGaugh, JL.Weinberger, N., Lynch, G., editors. Brain and memory: Modulation and mediation of neuroplasticity. New York: Oxford University Press; in press

Markowska AL, Stone WS, Ingram DK, Reynolds J, Gold PE, Conti LH, Pontevorvo MJ, Wenk GL, Olton DS. Individual differences in aging: Behavioral and neurobiological correlates. Neurobiology of Aging. 1989; 10:31-43. [PubMed: 2569170]

Morris RGM. Spatial localization does not require the presence of local cues. Learning and Motivation. 1981; 12:239-260.

Morris RGM, Garrud P, Rawlins JNP, O'Keefe J. Place navigation impaired in rats with hippocampal lesions. Nature. 1982; 297:681-683. [PubMed: 7088155]

Morris RGM, Schenk F, Tweedie F, Jarrard LE. Ibotenate lesions of hippocampus and/or subiculum: Dissociating components of allocentric spatial learning. European Journal of Neuroscience. 1990; 2:1016-1028. [PubMed: 12106063]

Nagahara AH, Nicolle MM, Gallagher M. Alterations in [3H]-kainate binding in the hippocampal formation of aged Long-Evans rats. Hippocampus. in press.

Olton, DS., Markowska, AL. The aging septohippocampal system: Its role in age-related memory impairments. In: Squire, L., Butters, N., editors. Neuropyschology of memory. New York: Oxford University Press; 1992. p. 378-385.

Rapp PR, Amaral DG. Individual differences in the behavioral and neurobiological consequences of normal aging. Trends in Neuroscience. 1992; 15:340-345.

Rapp P, Rosenberg R, Gallagher M. An evaluation of spatial information processing in aged rats. Behavioral Neuroscience. 1987; 101:3-12. [PubMed: 3828055] 

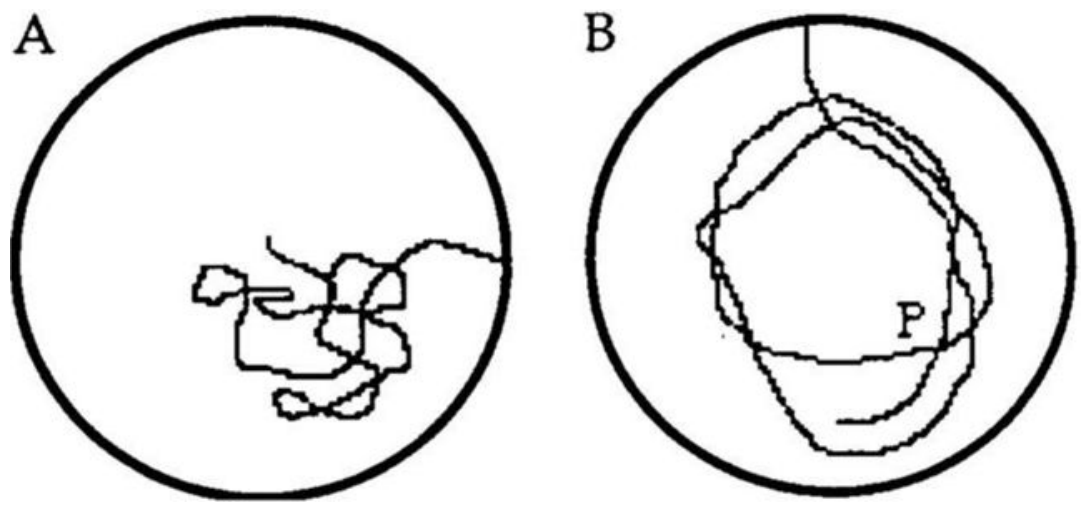

Figure 1.

Computer tracking from probe trials (30-s duration) in which the platform was unavailable for escape. (These probe trials occurred after 18 training trials [ 3 days at 6 trials/day]. The position of $\mathrm{P}$ identifies the quadrant in which the escape platform was located during training. The control rat [shown in A.] exhibits an appropriately focused search. The rat with hippocampal damage [shown in B.] swims in a concentric pattern at a relatively fixed distance from the wall. From "Preserved Configural Learning and Spatial Learning Impairment in Rats With Hippocampal Damage" by M. Gallagher and P. C. Holland, 1992, Hippocampus, 2, p. 85. Copyright 1992 by Churchill-Livingstone. Reprinted by permission.) 

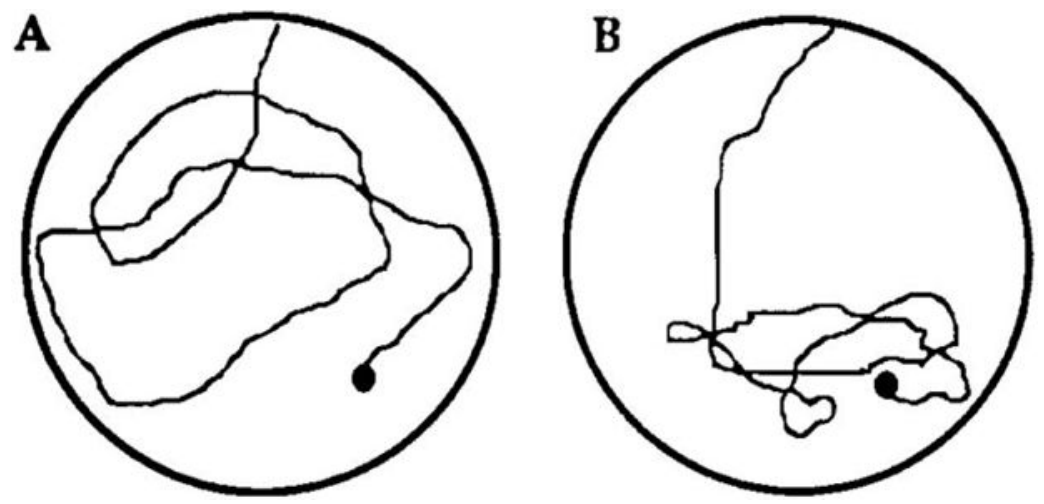

Figure 2.

Computer tracking of the paths taken by rats on two comparison training trials. (The path length in A is $573 \mathrm{~cm}$, compared with $590 \mathrm{~cm}$ for the record in B. In contrast to the similar path length for the two records, cumulative distance from the goal during the two trials differs substantially: 1,835 and $781 \mathrm{~cm}$ for A and B, respectively. In the calculation of cumulative distance, the animal's position in the maze was sampled in $\mathrm{x} / \mathrm{y}$ coordinates at a rate of 10 times per second. One-second averages of the rat's distance from the escape platform are summed to provide the cumulative distance measure provided above. Additional refinements to this basic procedure are described in the Method section.) 

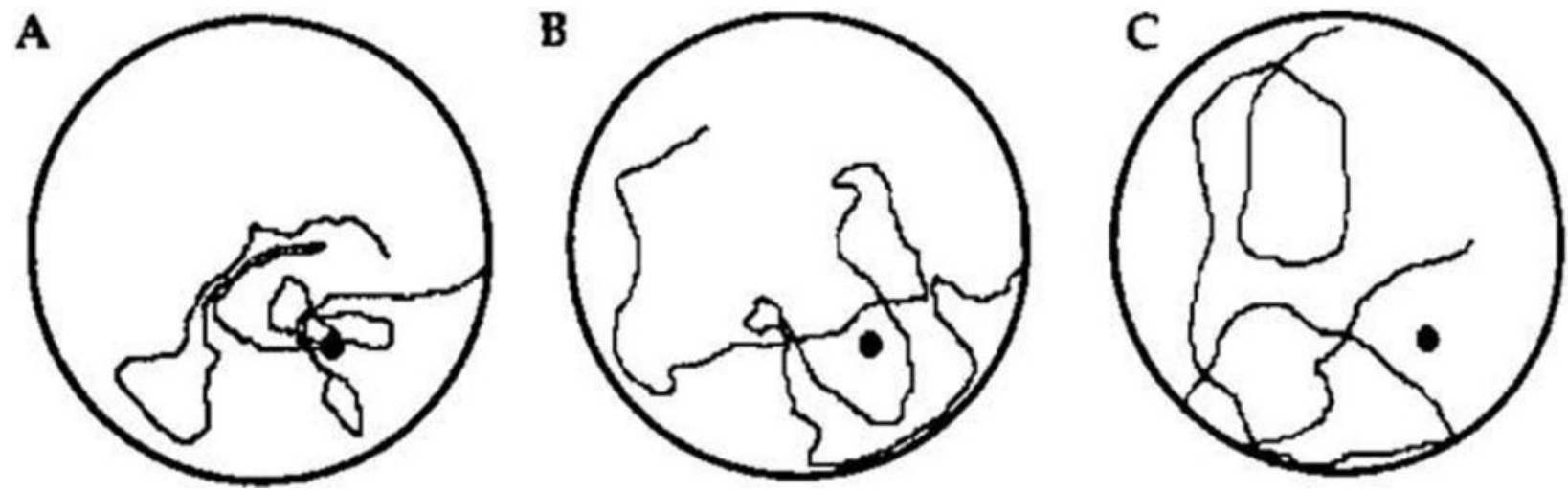

Figure 3.

Computer tracking of the paths taken on three sample 30-s probe trials. (The percentages of the path length in the training quadrant for $\mathrm{A}, \mathrm{B}$, and $\mathrm{C}$ were $52 \%, 49 \%$, and $15 \%$, respectively. The average distances from the platform during the search were 36.9, 50.7, and $75.2 \mathrm{~cm}$ for A, B, and C, respectively. Average proximity was calculated from 1-s averages that were obtained as described in the caption to Figure 2.) 


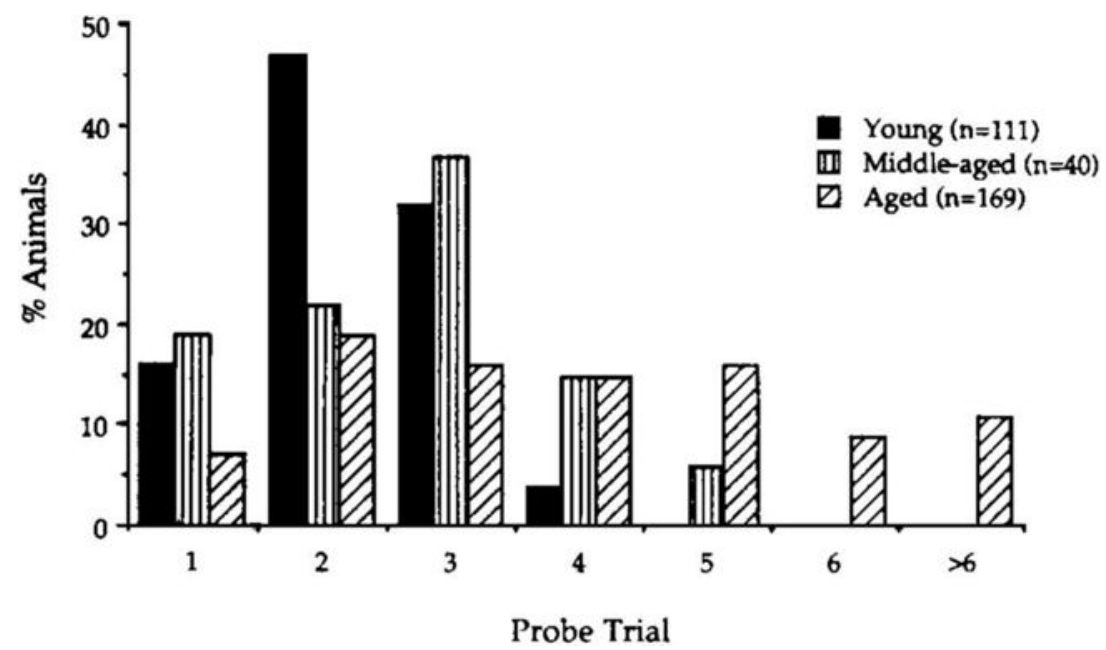

Figure 4.

Acquisition of criterion performance in a number of previous studies (Probe trials [30-s duration] were interpolated after each block of five training trials. Bars represent the percentage of animals in each group that initially reached criterion at each probe trial. Criterion was reached on a probe trial when $30 \%$ of the path length was traversed in the maze quadrant that contained the escape platform during training and at least two platform crossings occurred. 


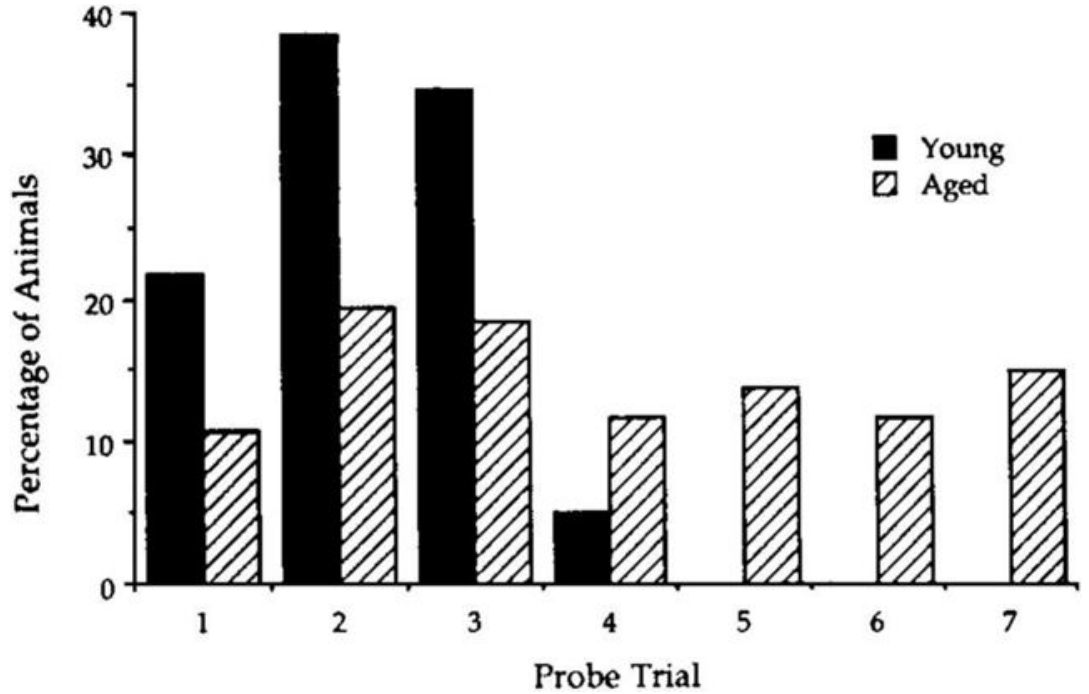

Figure 5.

Acquisition of criterion performance in the present study. (Bars represent the percentage of animals in each group that initially reached criterion at each probe trial. Criterion was reached on a probe trial when $35 \%$ of the duration of the trial was traversed in the maze quadrant that contained the escape platform during training and at least two platform crossings occurred.) 


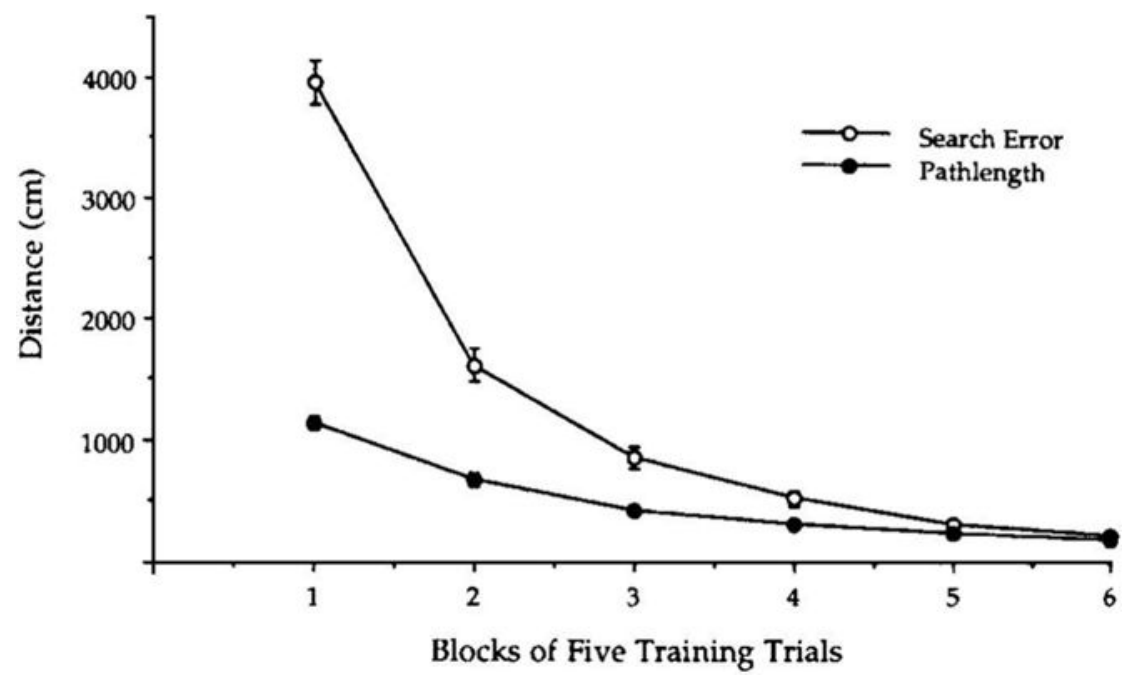

Figure 6.

Two measures obtained on training trials for the young group of rats. (Path length to locate the platform is compared with search error. Data points represent the average for blocks of five training trials. See text for results of statistical analysis.) 


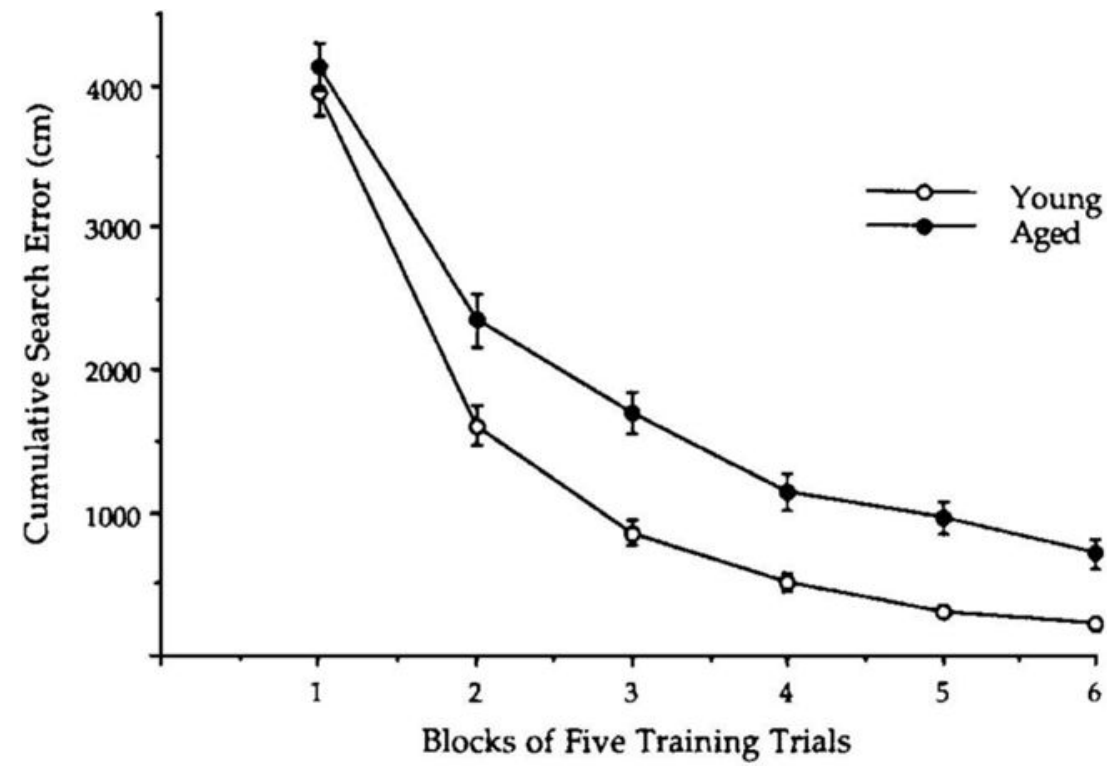

Figure 7.

The performance of young and aged groups during training trials. (Search error is the measure used. Data points are averages for blocks of five training trials. See text for results of statistical analysis.) 


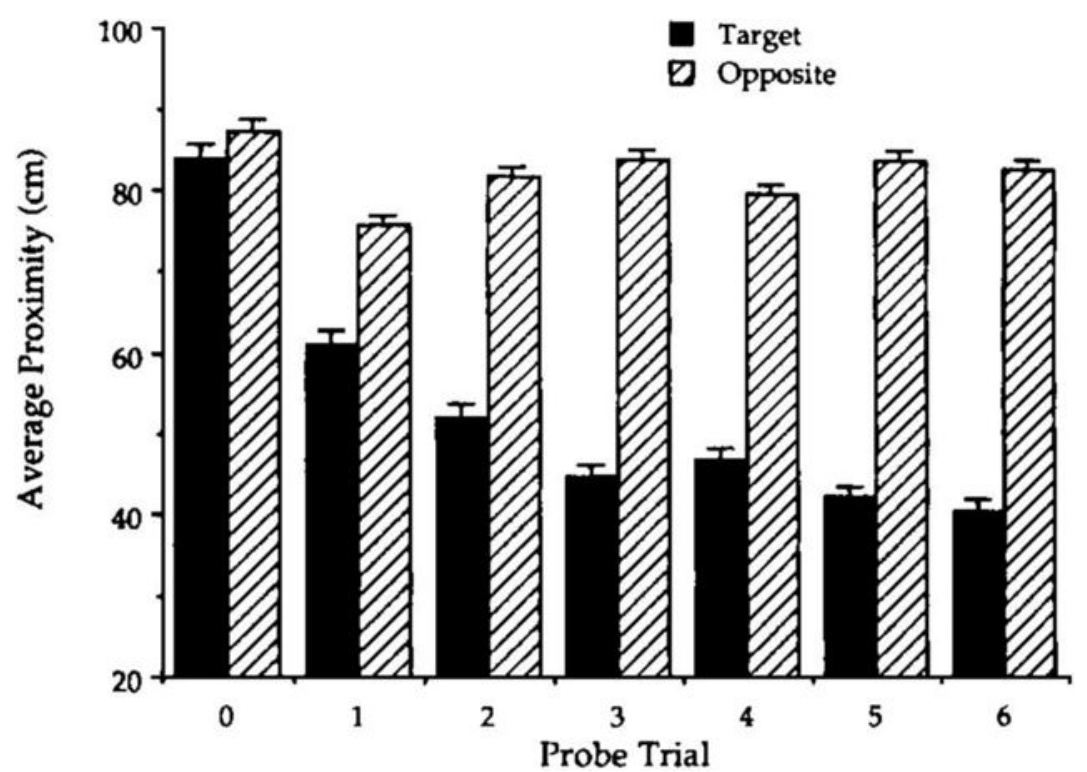

Figure 8.

Performance on probe trials (1-6) and for the first $30 \mathrm{~s}$ of the first training trial (designated Trial 0) for the young rats. (Distance from Target refers to the average distance from the escape platform location on the trial. Distance from Opposite refers to the average distance from a comparable location in the opposite quadrant of the maze, that is, $180^{\circ}$ from the target. See text for results of statistical analysis.) 


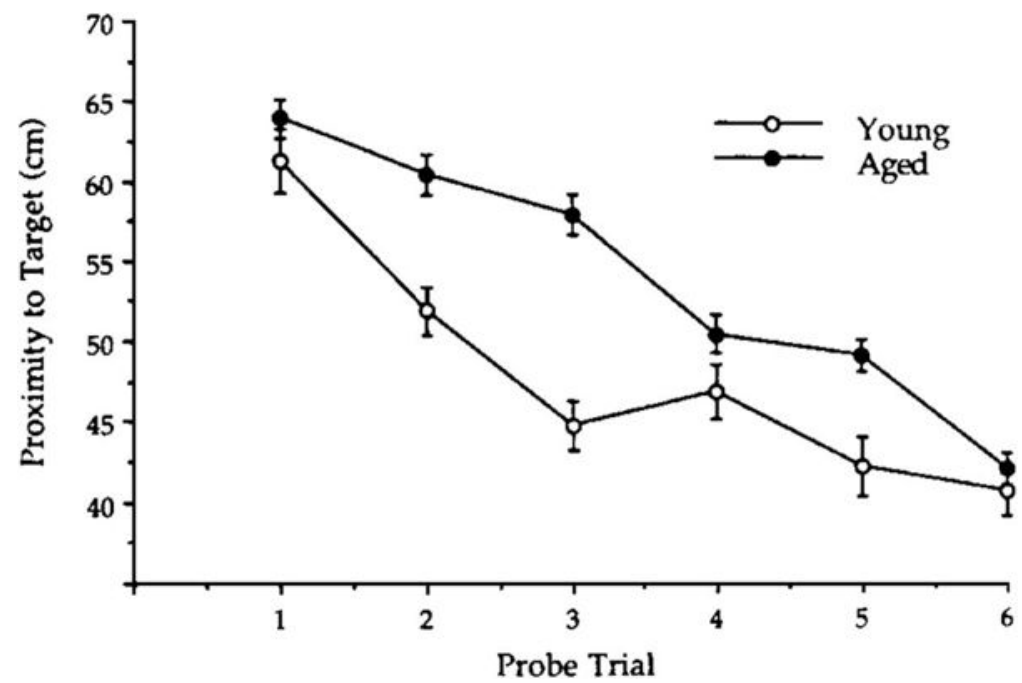

Figure 9.

Performance of the young and aged groups for the probe trials. (Data points represent the average distance from the target location. See text for results of statistical analysis.) 

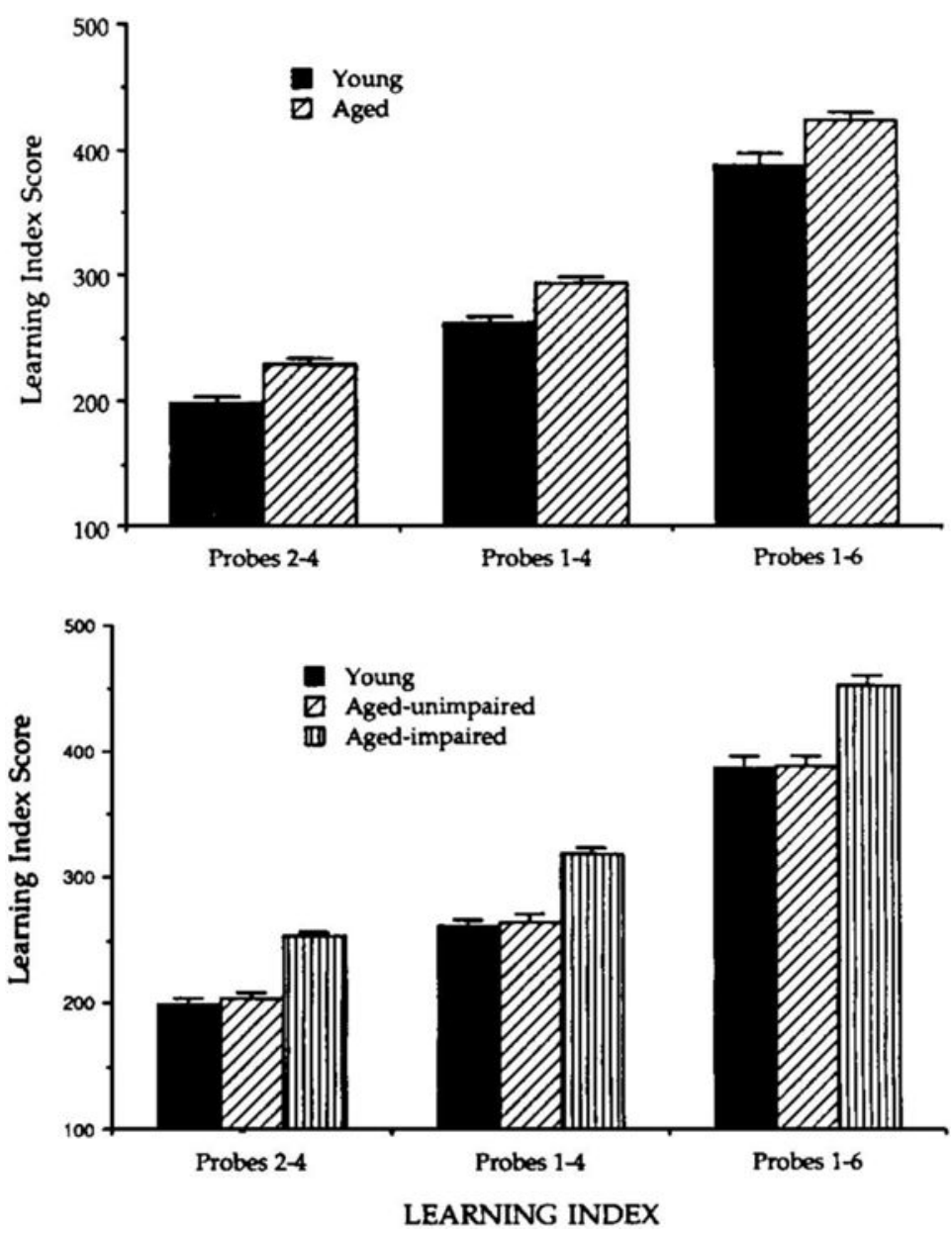

Figure 10.

Learning index based on probe trial performance. (In the upper panel, learning index scores are shown for young and aged groups. The three forms of the index were based on Probe Trials 1-6, 1-4, and 2-4, respectively. In the lower panel, the same data are shown with the aged rats grouped according to the criterion performance used in previous research [data shown in Figure 5]. See text for results of statistical analysis.) 


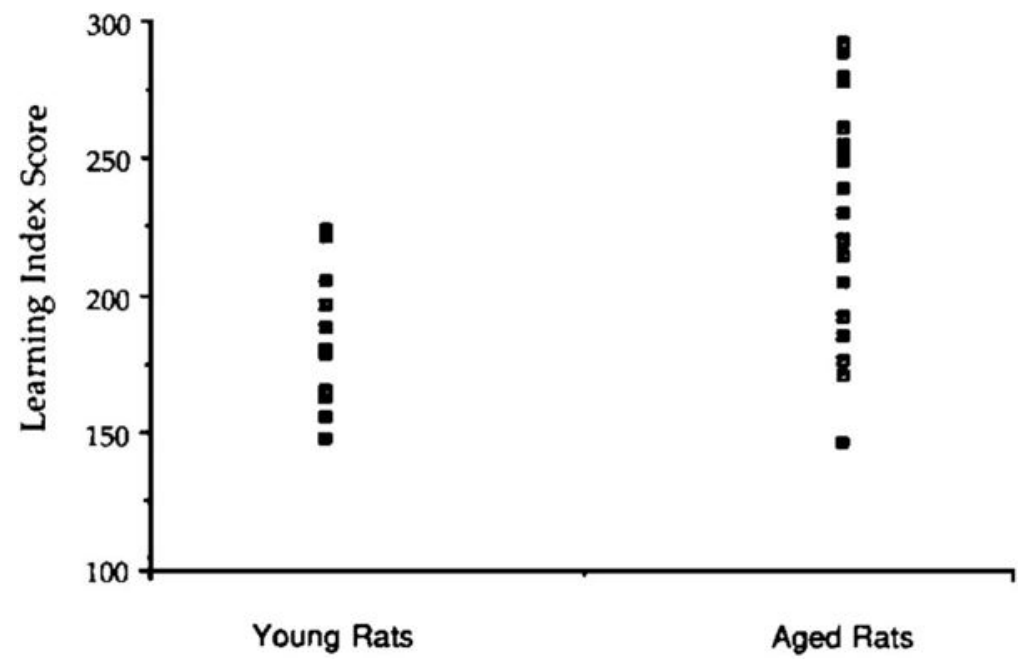

Figure 11.

Individual scores for the index based on Probe Trials 2-4 for one sample replication. (Within this replication, the scores for the young group differed significantly from those for the aged group, $\mathrm{p}<.01$. Reliable differences have been consistently found for index scores involving the numbers of animals typically used for age comparisons [see Burwell \& Gallagher, 1993; Nagahara, Nicolle, \& Gallagher, in press].) 


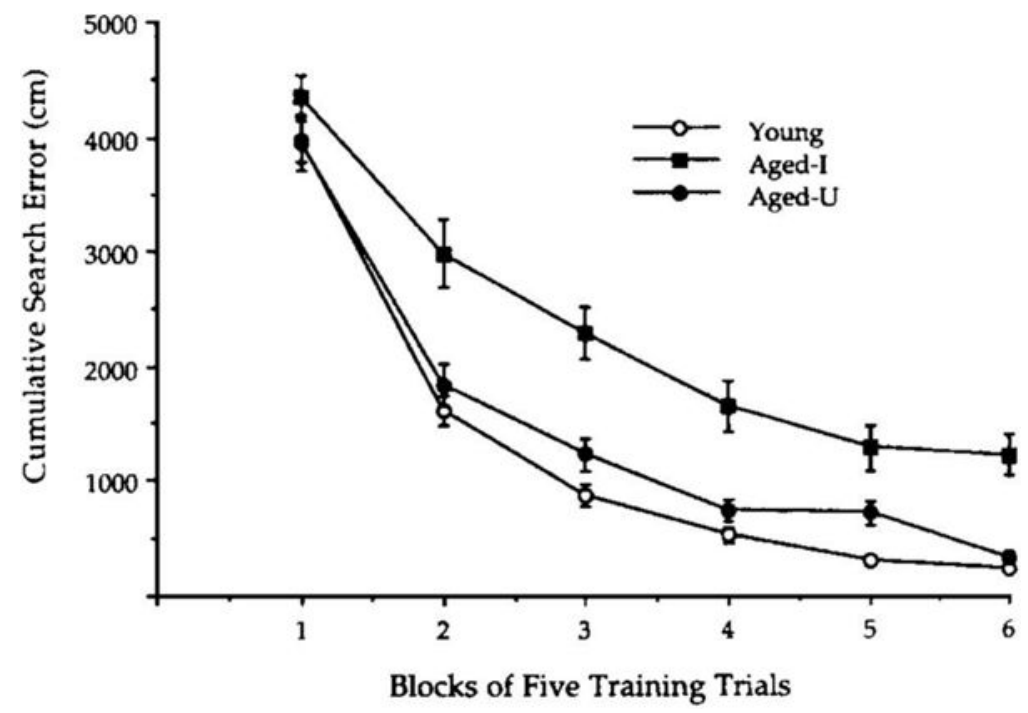

Figure 12.

Training trial performance (search error) for young rats (Young), aged rats that had scores of 240 or greater on the same index shown in Figure 11 (Aged-I), and aged rats that had index scores below 240 (Aged-U). (Means $[ \pm$ SEM] are shown for blocks of five training trials. See text for description of statistical analysis. $\mathrm{I}=$ impaired; $\mathrm{U}=$ unimpaired.) 

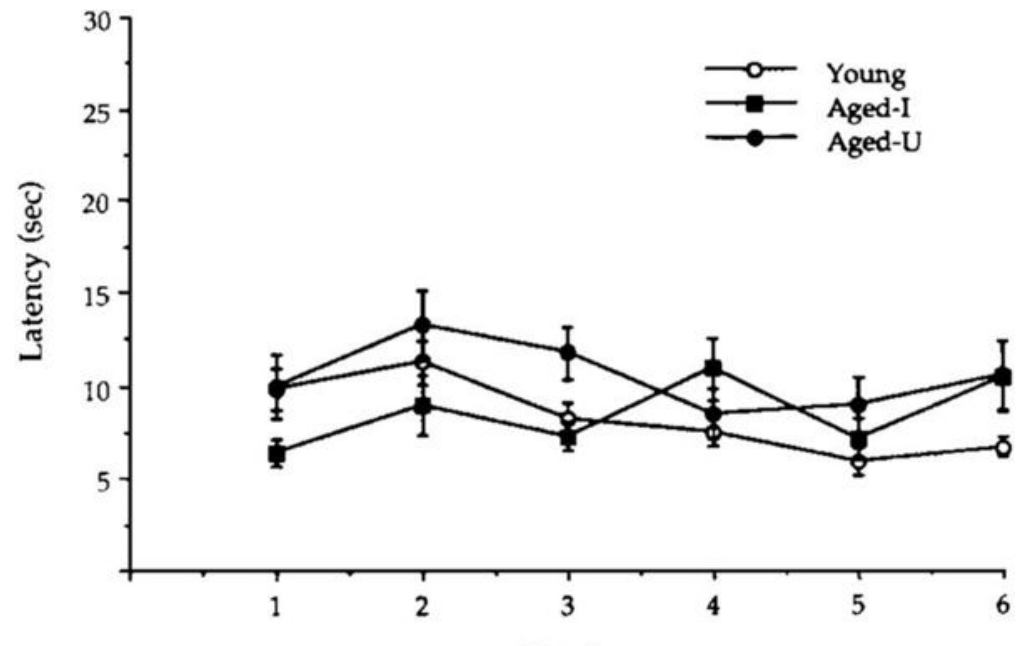

Figure 13.

Cue training performance (latency) for the groups described in Figure 12. (Means $[ \pm$ SEM] are for each trial in a session of six training trials. See text for description of statistical analysis. $\mathrm{I}=$ impaired; $\mathrm{U}=$ unimpaired.) 